\title{
VoIP-PSTN Interoperability by Asterisk and SS7 Signalling
}

\author{
Jan Rudinsky \\ CESNET, z. s. p. o. \\ Zikova 4, 16000 Praha 6, Czech Republic \\ rudinsky@cesnet.cz
}

\begin{abstract}
PSTN, the world's circuit-switched network, has employed Signalling System \#7 as its protocol suite for international and national interconnection during past decades. VoIP networks however have developed different signalling protocols suitable for IP environment. Gateways interconnecting VoIP and PSTN networks are usually proprietary and expensive solutions. Today an open source software can perform this function.

As an example we have decided to test Asterisk PBX and two open source implementations of SS7, the SS7 channel driver and SS7 library. We have tested these solutions for interconnection to PSTN and run various tests to verify the implementation functionality.
\end{abstract}

\section{Introduction}

\subsection{Signalling System \#7}

Common Channel Signalling System No.7 (also referred as SS7, CCS7 or C7) is a suit of control protocols used to provide instructions between elements within a public switched telephony network (PSTN). These instructions carry information about routing calls, requested services by subscribers, identities of participants and management information for signalling network.

Several organizations have written standards for SS7 networks. ITU-T standard is used on the international level of interconnection between signalling networks and it is the most common SS7 standard used on the national level in Europe. The national level of the network can generally use standard whatever exists within the country. An example is the ANSI standard used within the United States. We have tested the ITU variant of SS7 protocol suite as defined in ITU-T Q.7xx set of recommendations.

\subsection{Asterisk PBX}

Asterisk is a software private branch exchange (PBX). Unlike the traditional PBX being bound to vendor specific equipment, Asterisk can be run on almost any kind of hardware which uses Linux, BSD or Mac OS as their operating system. Asterisk supports functions such as traditional PBX services as well as set of additional functions including voice mail, interactive voice response or automatic call

Please use the following format when citing this chapter:

Rudinsky, J., 2007, in IFIP International Federation for Information Processing, Volume 245, Personal Wireless Communications, eds. Simak, B., Bestak, R., Kozowska, E., (Boston: Springer), pp. 169-173. 
distribution.

The system can interface traditional circuit-switched systems such as PSTN network as well as packet based systems represented by Voice over IP (VoIP) networks. For interconnection with VoIP networks Asterisk doesn't need any additional hardware. However for interconnection with PSTN networks and almost all standards-based telephony equipment an additional hardware

is necessary. These hardware devices come in a form of PCI cards with analog or digital telephony interfaces. Related protocol support by Asterisk covers wide range of VoIP signalling protocols such as Session initiation protocol (SIP), Inter Asterisk Exchange protocol (IAX), Media gateway control protocol (MGCP), H.323 and Cisco Skinny protocol. The TDM protocol group includes most of the European and American standard signalling types, the analog E\&M, FXO, FXS and multi frequency tones as well as the digital ISDN protocol group.

\section{Configuration}

\subsection{System Configuration}

Asterisk software PBX with SS7 support can be run on almost any kind of hardware. In this example we use Intel x386 family processor, standard network Ethernet adaptor and PCI bus controlled card with ISDN Primary Rate Interface (ISDN PRI) manufactured at Digium.

The system is based on GNU/Linux operating system, distribution Debian. The distribution includes device drivers handling the Ethernet card and other components. The PCI card specific driver has to be compiled from the source code given by the card manufacturer. Zaptel is a kernel interface device driver of Digium cards with analog or digital interface cards. The driver is then included in the system as a kernel loadable module.

Software requirements to install Asterisk application include following important packages:

$\begin{array}{ll}> & \text { linux } 2.6 \text { kernel headers } \\ & \text { bison and bison-dev } \\ & \text { ncurses and ncurses-dev } \\ & \text { zliblg and zlib1g-dev } \\ > & \text { libssl and libssl-dev } \\ > & \text { libnewt-dev } \\ & \text { initrd-tools, cvs and procps }\end{array}$

\subsection{Asterisk SS7 Installation and Configuration}

The Asterisk source code is available at the developers web page [1]. Important packages include "Asterisk" and "libpri", an ISDN PRI library for E1 interface. The installation is done by standard make - make install procedure.

Each signalling protocol is implemented in Asterisk as a channel, that represents incoming and outgoing protocol sessions. First signalling solution, the SS7 channel 
driver is implementated in a form of SS7 channel that process SS7 messages. SS7 channel driver includes implementations of MTP2 layer, bare essentials of MTP3 and a large subset of ISUP functions. It can be downloaded from the developers web page [2] and compiled into the Asterisk loadable module.

Second solution, the SS7 library makes use of standard Zaptel channel. This channel is a part of the Asterisk source code and is used to manage sessions via Zaptel compatible hardware cards with E1 interface.

Configuration of lowest protocol layers is stored in "zaptel.conf" and it is similar for both SS7 solutions. This file is read by zaptel driver, which accordingly sets the link encoding, timing source for synchronisation, type of error detection code and mapping of signalling and traffic channels.

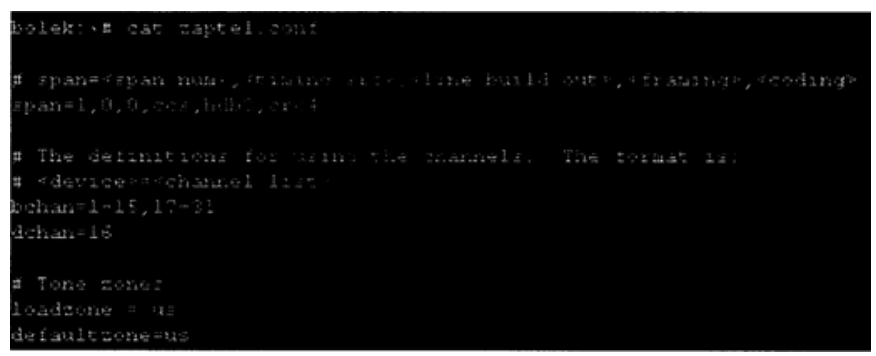

Fig. 1. Zaptel driver configuration

Higher layer configuration of SS7 message transfer part and user part is different for each solution. SS7 channel driver configuration is stored in file "ss7.conf". The content of the file defines SS7 signalling linkset properties, parameters of signalling links including signalling and traffic channel allocation and point code assignments. SS7 library configuration file zapata.conf contains similar parameters definition only in a different format.

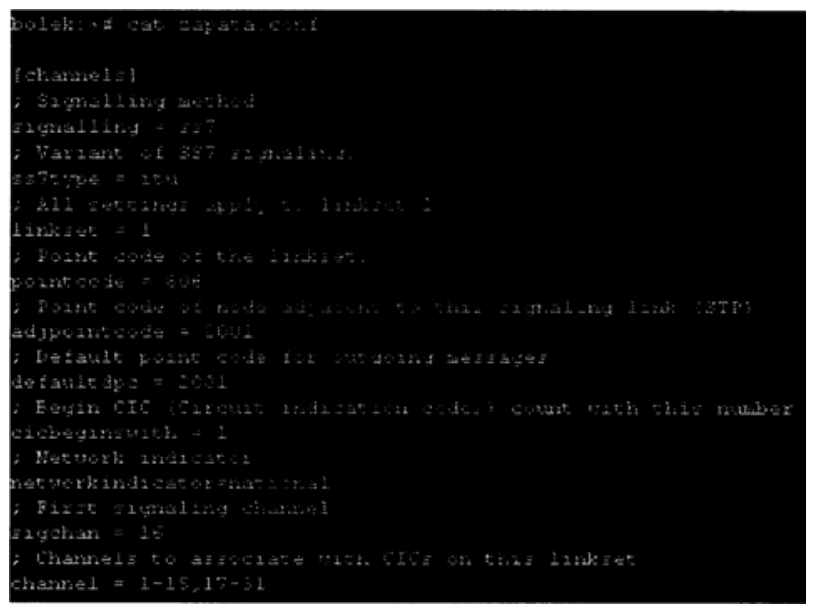

Fig. 2. SS7 higher layer configuration 
To define the Asterisk behaviour we have to set the Asterisk dialplan. Configuration file extensions.conf contains necessary information to control the execution of call flow and related operations.

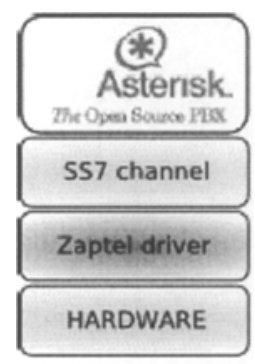

S57 channel driver

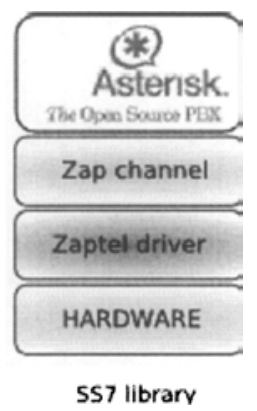

SS7 library

Fig. 3. Asterisk SS7 stack

\section{Signalling and Voice Traffic Tests}

We have verified the functionality of Asterisk PBX with SS7 support in various tests. In the first scenario we have used Tektronix K1297 protocol tester to test the SS7 message transfer part (MTP) layer implementation of SS7 channel driver. The tests ended with positive result leaving SS7 link "In service" and voice channels in "Idle" mode.

Second scenario was represented by two Asterisk boxes, each with one SS7 solution installed. The servers were interconnected via E1 crossover cable plugged into the Digium TE110P interface cards at each end. After the initial configuration and Asterisk startup we have manually loaded the SS7 channel and Zap channel drivers on both servers. The initial procedure of MTP alignment and a group reset of E1 circuits ran successfully and ISUP controlled calls were exchanged in both directions.

The next phase was focused on Asterisk to PSTN interconnection. The telephone network was represented by Ericsson AXE platform mobile switching center (MSC). We have used standard E1 interface with 30 traffic channels and a single SS7 signalling channel. We have tested subsequently both SS7 solutions. After solving initial problems with SPCs (Ericsson and SS7 library using decimal, while SS7 channel driver hexadecimal numbers) and Asterisk exception of counting Circuit Identification Codes from 0 , we have successfully interconnected the exchanges. Initialization process of the SS7 connection was identical to the previous case and successful outcome was confirmed by Asterisk on the console. We have passed calls in both directions.

Besides the basic call setup we have tested the SS7 channel driver also for several supplementary services. The implementation of calling line identity services presentation and restriction corresponds to the recommendations, while the connected line presentation and restriction seem not to be supported. The call waiting and call hold supplementary services were tested with successful outcome, while call forwarding was not supported. 


\section{Conclusions}

Signalling System \#7 is today's most common signalling protocol in the PSTN worldwide and a share of PSTN on the telecommunication market is large. However the market represented by VoIP networks is still growing and will eventually prevail PSTN networks. Therefore there is a need for interconnection between these types of network.

Current solutions are proprietary and usually expensive systems. As an option we can use Asterisk PBX as a gateway between VoIP and PSTN using Signalling System \#7. It represents an open source solution with wide range of supported protocols (SIP, MGCP, H.323) among which the SS7 has been added. The system is running on GNU/Linux operating system ensuring credible amount of reliability.

As the test have proven, both SS7 solutions, the SS7 channel driver and the SS7 library, can perform large subset of SS7 functionality including routing and call setup and a basic subset of supplementary service. Though some functions remain to be implemented.

Additionally Asterisk can perform as a media gateway by supporting number of codecs (G.711, G.723.1, G.726, G.729, GSM, iLBC, LPC-10 or Speex) and translation between them.

Acknowledgement. This project is carried out as a part of research of IP telephony group at CESNET z.s.p.o. CESNET is a Czech academic network operator with the mission of research in advanced network technologies and applications. The major framework for our research is the official research plan titled Optical National Research Network and its New Applications which is implemented since 2004 until 2010.

\section{References}

1. http://www.asterisk.org

2. http://www.sifira.dk/chan-ss7

3. T. Russell, "Signalling System \#7", McGraw-Hill (2002), ISBN 0-07-138772-2 